\title{
Analytical and numerical results of fractional differential-difference equations
}

\author{
Najeeb Alam Khan \\ Department of Mathematical Sciences, \\ University of Karachi, Pakistan
email: njbalam@yahoo.com \\ University of Karachi, Pakistan
email: njbalam@yahoo.com
}

\author{
Fatima Riaz \\ Department of Mathematical Sciences, \\ University of Karachi, Pakistan \\ email: fatima.riaz@ymail.com
}

\begin{abstract}
In this paper, we examine the fractional differential-difference equation (FDDE) by employing the proposed sensitivity approach (SA) and Adomian transformation method (ADTM). In SA the nonlinear differential-difference equation is converted to infinite linear equations which have a wide criterion to solve for the analytical solution. By ADTM, the FDDE is converted into ordinary differential-difference equation that can be solved. We test both the techniques through some test problems which are arising in nonlinear dynamical systems and found that ADTM is equivalently appropriate and simpler method to handle than SA.
\end{abstract}

\section{Introduction}

A differential-difference equation (DDEs), first studied by Fermi et al. [1] in the 1950s is of enormous significance in describing physical phenomena of various fields such as mechanical engineering, biophysics, condensed matter physics, and in different physical problems like currents flow in electrical networks [2], particle vibrations in lattices, and pulses in biological chains [3]. Many forms of DDEs are discovered to analyze the discrete nonlinear system.

2010 Mathematics Subject Classification: 37N30, 34A08

Key words and phrases: differential-difference equation, transformation, sensitivity approach, numerical solutions 
In recent decades fractional derivatives are introduced to deal with non differentiable functions. The theory of using integrals and derivatives of an arbitrary order, fractional calculus, discussed about 300 years ago, have applications in fractional control of engineering systems, acoustics, damping laws, bioengineering and biomedical applications, electromagnetism, hydrology, signal processing, and many others $[4,5,6]$. It is used for examining stochastic processes forced by fractional Brownian processes [7, 8], non-random fractional phenomena in physics $[9,10,11]$, the study of porous systems, and quantum mechanics $[12,13]$.

Recently, there have been a number of schemes committed to the solution of fractional differential equations. The Adomian decomposition method [14], homotopy perturbation method $[15,16,17]$, homotopy analysis method [18, 19], Taylor matrix method [20] and many others have been used to solve the fractional differential equations.

In the present paper, the sensitivity approach $[21,22,23]$ which has been presented to solve various kinds of optimal control problems and analysis of time delay systems has used. In this approach, a sensitivity parameter has introduced which transform the original nonlinear fractional differential-difference equation (FDDE) to a linear sequence of FDDEs. The system of equations then now consists of a linear term and a nonlinear series terms. Iterations have been done only for nonlinear series terms, i.e., the result of a sequence of linear FDDEs leads to nonlinear terms for compensation is extended to solve FDDEs. Also, the Adomian decomposition method (ADM) has been modified by a special transformation. The transformation has converted the fractional order differential-difference equation to ordinary differential-difference equation which then solved by the Adomian decomposition method.

\section{Preliminaries}

The modified Riemann-Liouville derivative of order $\alpha$ is defined, for a function $f(x)$, by

$$
\begin{aligned}
& D_{x}^{\alpha} f(x)=\frac{1}{\Gamma(-\alpha)} \int_{0}^{x}(x-\eta)^{-1-\alpha}(f(\eta)-f(0)) d \eta ; \quad \alpha<0, \\
& D_{x}^{\alpha} f(x)=\frac{1}{\Gamma(1-\alpha)} \frac{d}{d x} \int_{0}^{x}(x-\eta)^{-\alpha}(f(\eta)-f(0)) d \eta ; \quad 0<\alpha<1, \\
& D_{x}^{\alpha} f(x)=\left(f^{(n)}(x)\right)^{(\alpha-n)} d \eta ; \quad n \leq \alpha \leq n+1, \quad n \geq 1,
\end{aligned}
$$


where $f: \mathbf{R} \rightarrow \mathbf{R}$ is a continuous function. Properties of modified Riemann Liouville derivative are given as

$$
\begin{aligned}
& D_{\chi}^{\alpha} x^{(\beta)}=\frac{\Gamma(\beta+1)}{\Gamma(\beta-\alpha+1)} x^{\beta-\alpha} ; \quad \beta>0, \\
& d^{\alpha} x(t)=\Gamma(\alpha+1) d x(t) ; \quad \beta>0,
\end{aligned}
$$

\section{Implementation of the methods}

Consider the nonlinear FDDEs in the form of:

$$
D_{t}^{\alpha} U_{n}(t)=\mathcal{N}\left(\ldots, u_{n-1}(t), u_{n}(t), u_{n+1}(t), \ldots\right) ;
$$

with initial conditions

$$
\mathrm{u}_{\mathrm{n}}\left(\mathrm{t}_{0}\right)=f(\mathrm{n})
$$

where $\mathcal{N}$ is the non linear function, $\mathrm{U}_{\mathrm{n}}(\mathrm{t})$ is an unknown function, $f(\mathrm{n})$ is the initial condition and $t, n$ are independent variables. Assuming that a unique solution exist for Eq. (4). It is difficult to obtain exact solution of nonlinear FDDE (6). Only in some cases, we can obtain exact solution.

\section{Sensitivity approach (SA)}

In this approach, a sensitivity parameter $\Lambda$, which varies between zero and unity, is introduced into nonlinear terms of FDDE. When $\Lambda=0$, the nonlinear problem is transformed to a simple problem, which can be solved through analytic method. When $\Lambda=1$, the original nonlinear problem is obtained. This transformation leads to solving a sequence of linear FDDEs instead of solving nonlinear FDDEs. Now, we embedded a sensitivity parameter $\Lambda$ in Eqs. (6)-(7) and form the following sensitized FDDEs:

$$
D_{t}^{\alpha} u_{n}(t, \Lambda)=\mathcal{N}\left(\ldots, u_{n-1}(t, \Lambda), u_{n}(t, \Lambda), u_{n+1}(t, \Lambda), \ldots\right)
$$

with initial conditions

$$
\left.\mathrm{u}_{\mathrm{n}}(\mathrm{t}, \Lambda)\right|_{\mathrm{t}=\mathrm{t}_{0}}=f(\mathrm{n})
$$

Where $0 \leq \Lambda \leq 1$. In the following explanation, we suppose that the solution of Eq. (6) is distinctively existed and $U_{n}(t, \Lambda)$ with $\Lambda$ is infinitely differentiable 
with respect to the $\Lambda$ in the region of $\Lambda=0$, and its Maclaurin series is convergent at $\Lambda=1$. Apparently when $\Lambda=1 \mathrm{Eq}$. (8) is equivalent to the original problem Eq. (6). According to the assumption we can write:

$$
\mathrm{u}_{n}(\mathrm{t}, \Lambda)=\sum_{n=1}^{\infty} \Lambda^{j} \mathrm{u}_{\mathrm{n}}^{(\mathrm{j})}(\mathrm{t})
$$

where $(*)^{(j)}=\left.\frac{1}{j !} \frac{\partial^{j}}{\partial \Lambda^{j}}(*)\right|_{\Lambda=0}$ Now, we substitute Eq. (10) into Eq. (8) and equating terms with the same order of $\Lambda$ on each side we have:

$$
\begin{gathered}
\Lambda^{0}: D_{t}^{\alpha} u_{n}^{(0)}(t)=\mathcal{N}\left(u_{n}^{(0)}(t)\right), \quad u_{n}^{(0)}\left(t_{0}\right)=f(n), \\
\Lambda^{1}: D_{t}^{\alpha} U_{n}^{(1)}(t)=\mathcal{N}\left(U_{n}^{(1)}(t)\right)+g_{n}^{(0)}(t), \quad u_{n}^{(1)}\left(t_{0}\right)=0, \\
\Lambda^{j}: D_{t}^{\alpha} u_{n}^{(j)}(t)=\mathcal{N}\left(u_{n}^{(j)}(t)\right)+g_{n}^{(j-1)}(t), \quad U_{n}^{(j)}\left(t_{0}\right)=0,
\end{gathered}
$$

Where, $g^{(j-1)}(t)$ is the coefficient of $\Lambda^{(j-1)}$ in the expanding of $f(n)$ and can be resolve in the following manner:

$$
g^{(j-1)}(t)=\left.\frac{1}{(j-1) !} \frac{\mathcal{N}\left(\ldots, u_{n-1}(t, \Lambda), u_{n}(t, \Lambda), u_{n+1}(t, \Lambda), \ldots\right)}{\partial \Lambda^{(j-1)}}\right|_{\Lambda=0},
$$

It should be noticed that Eq. (11) gives linear approximate and Eq. (6) gives correction term to linear approximate solution by keeping in consideration second order nonlinearity and so on. If the above process caries on, at each step, a system of non-homogeneous linear FDDEs is obtained in which nonhomogeneous terms are known from the previous step. Hence, solving the presented sequence is a recursive process. After indentifying $U^{j}(t)$ for $j \geq 1$, it is obvious that $\Lambda=1$ should be set in Eq. (8) and Eq. (9) so that they deform to the exact solution of Eq. (6) and so we have:

$$
\mathrm{u}_{n}(\mathrm{t}, 1)=\sum_{j=0}^{\infty} \mathrm{u}_{\mathrm{n}}^{(\mathrm{j})}(\mathrm{t})
$$

In this way, the original nonlinear FDDE has been converted into a sequence of linear FDDEs, which should be solved in a recursive development and this will overcomes the complexity of working with nonlinear FDDEs. It is clear from the above procedure, a nonlinear FDDE is transformed into a sequence 
of linear FDDEs. In order to solve the Eq. (8), the following sensitized linear FDDE can be constructed:

$$
\begin{gathered}
D_{t}^{\alpha} U_{n}^{(0)}(t, \Lambda)=\Lambda \mathcal{N}\left(\ldots, u_{n-1}^{(0)}(t, \Lambda), u_{n}^{(0)}(t, \Lambda), u_{n+1}^{(0)}(t, \Lambda), \ldots\right) \\
u_{n}^{(0)}(t, \Lambda)=f(n)
\end{gathered}
$$

Assuming the solution of Eq. (16) as

$$
\mathrm{u}_{\mathrm{n}}(\mathrm{t}, \Lambda)=\sum_{n=1}^{\infty} \Lambda^{\mathrm{j}} \mathrm{u}_{\mathrm{n}}^{(0, j)}(\mathrm{t})
$$

Now by substituting Eq. (18) into Eq. (16) and equating terms with the same order of $\Lambda$ on each side we have:

$$
\begin{aligned}
& \Lambda^{0}: D_{t}^{\alpha} u_{n}^{(0,0)}(t)=\mathcal{N}\left(\ldots u_{n-1}^{(0,0)}(t, \Lambda), u_{n}^{(0,0)}(t, \Lambda), u_{n+1}^{(0,0)}(t, \Lambda), \ldots\right),(19) \\
& \mathrm{u}_{\mathrm{n}}^{(0,0)}\left(\mathrm{t}_{0}\right)=f(\mathrm{n}) \\
& \Lambda^{1}: D_{t}^{\alpha} u_{n}^{(0,1)}(t)=\mathcal{N}\left(\ldots u_{n-1}^{(0,1)}(t, \Lambda), u_{n}^{(0,1)}(t, \Lambda), u_{n+1}^{(0,1)}(t, \Lambda), \ldots\right),(20) \\
& \mathrm{u}_{\mathrm{n}}^{(0,1)}\left(\mathrm{t}_{0}\right)=0 \text {, } \\
& \Lambda^{j}: D_{t}^{\alpha} u_{n}^{(0, j)}(t)=\mathcal{N}\left(\ldots u_{n-1}^{(0, j)}(t, \Lambda), u_{n}^{(0, j)}(t, \Lambda), u_{n+1}^{(0, j)}(t, \Lambda), \ldots\right), \\
& u_{n}^{(0, j)}\left(t_{0}\right)=0 \text {, }
\end{aligned}
$$

By taking the inverse operator of $D_{t}^{\alpha}$ on applying Eqs. (19)-(21), we get the solution of FDDE as

$$
u_{n}^{0}(t)=u_{n}^{(0,1)}(t)=\sum_{j=0}^{\infty} u_{n}^{(0, j)}(t)
$$


In the same way, Eq. (6) has to be solved for $U_{n}^{0}(t)$ for $j \geq 1$. After some similar calculation we have:

$$
\begin{aligned}
& u_{n}^{j, k}(t)=u_{n}^{(j, 0)}(t)+u_{n}^{(j, 1)}(t)+u_{n}^{(j, 2)}(t)+u_{n}^{(j, 3)}(t)+\ldots \\
& u_{n}^{(j, 0)}(t)=0 \\
& \quad \vdots \\
& u_{n}^{(j, 1)}(t)=-J_{1}^{\alpha} g_{n}^{j-1}(t) \\
& \quad \vdots \\
& u_{n}^{(j, k)}(t)=-J_{1}^{\alpha} \mathcal{N}\left(\ldots u_{n-1}^{(j, k)}(t), u_{n}^{(j, k)}(t), u_{n+1}^{(j, k)}(t) \ldots\right)^{j} .
\end{aligned}
$$

Since, the steps above are enough to find the analytical solution, however, only some iteration of sub-problems and the original problem are sufficient to get a satisfactory accurate solution. We can replace $\infty$ by any positive integers $S$ and $T$ in the above mentioned series which may help in obtaining an approximate closed-form solution

$$
\mathrm{u}_{\mathrm{n}}(\mathrm{t})=\sum_{\mathrm{j}=0}^{S} \sum_{\mathrm{k}=0}^{T} \mathrm{u}_{\mathrm{n}}^{(\mathrm{j}, \mathrm{k})}(\mathrm{t})
$$

\section{Adomian decomposition transformation method (ADTM)}

Fractional complex transforms [24, 25] has now been become a useful tool to convert fractional differential equations to ordinary differential equations, which provides a very simple and easy solution approach. In the present method, the FDDE has transformed into ordinary DDE and then utilizing the Adomian decomposition method to solve for exact or analytic solutions. Duan $[26,27]$ has provided the efficient recurrence one variable formula to decompose the multivariable Adomian polynomials to solve the non-linear differential equation. Recalling the Eq. (6)

$$
D_{t}^{\alpha} u_{n}(t)=\mathcal{N}\left(\ldots, u_{n-1}(t), u_{n}(t), u_{n+1}(t), \ldots\right)
$$

with initial conditions

$$
\mathrm{u}_{\mathrm{n}}\left(\mathrm{t}_{0}\right)=f(\mathrm{n})
$$

Let us suppose

$$
\mathrm{T}=\frac{\mathrm{t}^{\alpha}}{\Gamma(\alpha+1)}
$$


Differentiating Eq. (28) and making use of modified Riemann Liouville derivative

$$
\frac{d^{\alpha} U_{n}(t)}{d t^{\alpha}}=U_{n}^{\prime}(T)
$$

Putting in Eq. (26), which transform the FDDE into ordinary DDE

$$
\mathrm{U}_{n, m}^{\prime}(\mathrm{T})=\mathcal{L} \mathrm{U}_{n, m}(\mathrm{~T})=\mathcal{N}\left(\ldots, \mathrm{U}_{n-1, m}(\mathrm{~T}), \mathrm{U}_{n, m}(\mathrm{~T}), \mathrm{U}_{\mathrm{n}+1, \mathrm{~m}}(\mathrm{~T}), \ldots\right)
$$

where $\mathcal{L}=\frac{\mathrm{d}}{\mathrm{dT}}$ and $\mathcal{L}^{-1}=\int_{\mathrm{T}_{\mathrm{o}}}^{\mathrm{T}}(*) \mathrm{dT}$ are the linear operator the inverse operators respectively. Applying the inverse operator on both sides of the Eq. (26) with Eq. (27) gives

$$
\mathrm{U}_{n, m+1}(\mathrm{~T})=f(\mathrm{n})+\mathcal{L}^{-1}\left[\mathcal{N}\left(\ldots, \mathrm{U}_{n-1, m}(\mathrm{~T}), \mathrm{U}_{n, m}(\mathrm{~T}), \mathrm{U}_{n+1, m}(\mathrm{~T}), \ldots\right)\right]
$$

In this section, three examples have presented to demonstrate the applicability of the suggested methods to solve nonlinear fractional differential-difference equations.

\section{Test problems}

\section{Problem 1}

Consider the following Volterra equation

$$
D_{t}^{\alpha} U_{n}(t)=U_{n}(t)\left(U_{n+1}(t)-U_{n-1}(t)\right)
$$

with initial conditions

$$
\mathrm{U}_{\mathrm{n}}(0)=\mathrm{n}
$$

The exact solution for $\alpha=1$ can be written as

$$
\mathrm{u}_{\mathrm{n}}(\mathrm{t})=\frac{\mathrm{n}}{(1-2 \mathrm{t})}
$$

For solving this equation, the following new equation is constructed with sensitivity parameter:

$$
D_{t}^{\alpha} U_{n}(t, \Lambda)=\Lambda U_{n}(t, \Lambda)\left(U_{n+1}(t, \Lambda)-U_{n-1}(t, \Lambda)\right)
$$

with initial conditions

$$
\mathrm{u}_{\mathrm{n}}(0, \Lambda)=\mathrm{n}
$$


Now assume:

$$
\begin{gathered}
u_{n}(t, \Lambda)=\sum_{j=0}^{\infty} \Lambda^{j} u_{n}^{(0, j)}(t) \\
u_{n+1}(t, \Lambda)=\sum_{j=0}^{\infty} \Lambda^{j} u_{n+1}^{(0, j)}(t) \\
u_{n-1}(t, \Lambda)=\sum_{j=0}^{\infty} \Lambda^{j} u_{n-1}^{(0, j)}(t)
\end{gathered}
$$

Substitute Eq. (36)-(38) in Eq. (34), it has been seen that the nonlinear original FDDEs are changed into a set of linear recursive FDDEs by using Eq. (19)-(21), in which at each step, the non-homogeneous terms are calculated from the preceding steps and process can be handled very simply which can solved the equation, we get the following series solution.

$$
\mathrm{u}_{n}(\mathrm{t})=\mathrm{n}+\frac{2 n \mathrm{t}^{\alpha}}{\Gamma(\alpha+1)}+\frac{8 n \mathrm{t}^{2 \alpha}}{\Gamma(2 \alpha+1)}+\frac{8 n t^{3 \alpha}\left(4 \Gamma(\alpha+1)^{2}+\Gamma(2 \alpha+1)\right)}{\Gamma(\alpha+1)^{2} \Gamma(3 \alpha+1)}+\ldots
$$

By putting $\alpha=1$, we recover ref. [24].

Now for the Adomian decomposition transformation method, Let us suppose

$$
\eta=\frac{1}{\Gamma(\alpha+1)} t^{\alpha}
$$

From modified Riemann Liouville derivative, we have

$$
\frac{d^{\alpha} U_{n}(t)}{d t^{\alpha}}=u_{n}^{\prime}(\eta)
$$

Using Eq. (41) in Eq. (32)

$$
\begin{gathered}
u_{n, m}^{\prime}=U_{n, m}(\eta)\left[u_{n+1, m}(\eta)-U_{n-1, m}(\eta)\right] \\
L U_{n, m}=A\left(u_{n, m}, U_{n+1, m}\right)-B\left(U_{n, m}, U_{n-1, m}\right)
\end{gathered}
$$

Operating $L^{-1}$ on both sides gives:

$$
\mathrm{U}_{n, m+1}=\mathrm{U}_{n}(0)+L^{-1}\left(A\left(\mathrm{U}_{n, m}, \mathrm{U}_{n+1, m}\right)-B\left(\mathrm{U}_{n, m}, \mathrm{U}_{n-1, m}\right)\right)
$$


Where the nonlinear terms $A\left(U_{n, m}, U_{n+1, m}\right)$ and $B\left(U_{n, m}, U_{n-1, m}\right)$ can be decomposed as follows:

$$
\begin{gathered}
A_{n, m}=\sum_{i=0}^{m-1} u_{n, i} u_{n+1, m-1-i} \quad B_{n, m}=\sum_{i=0}^{m-1} u_{n, i} u_{n-1, m-1-i} \\
A_{n, 1}=u_{n, 0} u_{n+1,0}, \quad B_{n, 1}=u_{n, 0} u_{n-1,0} \\
A_{n, 2}=u_{n, 1} u_{n+1,0}+u_{n, 0} u_{n+1,1}, \quad B_{n, 2}=u_{n, 1} u_{n-1,0}+u_{n, 0} u_{n-1,1} \\
A_{n, 3}=u_{n, 2} u_{n+1,0}+u_{n, 1} u_{n+1,1}+u_{n, 0} u_{n+1,2} \\
B_{n, 3}=u_{n, 2} u_{n-1,0}+u_{n, 1} u_{n-1,1}+u_{n, 0} u_{n-1,2}, \\
A_{n, 4}=U_{n, 3} u_{n+1,0}+u_{n, 2} u_{n+1,1}+u_{n, 1} u_{n+1,2}+u_{n, 0} u_{n+1,3} \\
B_{n, 4}=u_{n, 3} u_{n-1,0}+u_{n, 2} u_{n-1,1}+u_{n, 1} u_{n-1,2}+u_{n, 0} u_{n-1,3}
\end{gathered}
$$

The solution of the transformed problem is

$$
u_{n}=2 n \eta+4 n \eta^{2}+8 n \eta^{3}+16 n \eta^{4}+32 n \eta^{5}+64 n \eta^{6}+\ldots
$$

Now, replacing

$$
\eta=\frac{1}{\Gamma(\alpha+1)} t^{\alpha}
$$

in Eq.(46), we get

$$
\begin{aligned}
u_{n} & =2 n \frac{t^{\alpha}}{\Gamma(\alpha+1)}+4 n\left(\frac{t^{\alpha}}{\Gamma(\alpha+1)}\right)^{2}+8 n\left(\frac{t^{\alpha}}{\Gamma(\alpha+1)}\right)^{3} \\
& +16 n\left(\frac{t^{\alpha}}{\Gamma(\alpha+1)}\right)^{4}+\ldots
\end{aligned}
$$


Table 1: Numerical comparison of problem 1 at $t=0.01$

\begin{tabular}{|c|c|c|c|c|c|c|c|}
\hline $\mathrm{n}$ & $\alpha=0.5$ & & $\alpha=0.75$ & & & $\alpha=1$ & \\
\hline & SA & ADTM & SA & ADTM & SA & ADTM & Exact \\
-20 & -27.1982 & -25.8288 & -21.5089 & -21.4780 & -20.4082 & -20.4082 & -20.4082 \\
-10 & -13.5991 & -12.9144 & -10.7544 & -10.7390 & -10.2041 & -10.2041 & -10.2041 \\
10 & 13.5991 & 12.9144 & 10.7544 & 10.7390 & 10.2041 & 10.2041 & 10.2041 \\
20 & 27.1982 & 25.8288 & 21.5089 & 21.4780 & 20.4082 & 20.4082 & 20.4082 \\
\hline
\end{tabular}

\section{Problem 2}

Let us consider hybrid nonlinear difference equation of the Korteweg-de Vries $(\mathrm{KdV})$ equations

$$
D_{t}^{\alpha} U_{n}(t)=\left(1-\left(U_{n}(t)\right)^{2}\right)\left(U_{n+1}(t)-U_{n-1}(t)\right)
$$

with initial conditions

$$
\mathrm{U}_{\mathrm{n}}(0)=\tanh (\mathrm{k}) \tanh (\mathrm{kn})
$$

The exact solution of Eq. (48) for can be written as:

$$
\mathrm{U}_{\mathrm{n}}(\mathrm{t})=\tanh (\mathrm{k}) \tanh (\mathrm{kn}+2 \tanh (\mathrm{k}) \mathrm{t})
$$

Eq. (48) can be simplified as follows:

$$
D_{t}^{\alpha} U_{n}(t)=\left(U_{n+1}(t)-U_{n-1}(t)\right)-\left(u_{n}(t)\right)^{2} U_{n+1}(t)+\left(u_{n}(t)\right)^{2} u_{n-1}(t)
$$

The solution of Eq. (48) by SA is given by

$$
\begin{gathered}
\mathrm{u}_{\mathrm{n}}(\mathrm{t})=\tanh (\mathrm{k}) \tanh (\mathrm{kn})+\frac{1}{\Gamma(\alpha+1)} \mathrm{t}^{\alpha}(\cosh (2 \mathrm{k})+\cosh (2 \mathrm{kn}))\left(\frac{1}{\cosh (\mathrm{kn}-\mathrm{k})}\right) \\
\left.\left.\left(\frac{1}{(\cosh (k n))^{2}}\right)\left(\frac{1}{\cosh (k n+k)}\right)(\tanh (k))^{2}\right)\right)+\ldots
\end{gathered}
$$

Now, applying the Adomian transformation method the solution of Eq. (48) with Eq. (49) is

$\mathrm{u}_{n}=\tanh (k) \tanh (k n)+\eta(\cosh (2 k)+\cosh (2 k n))\left(\frac{1}{\cosh (k n-k)}\right)\left(\frac{1}{(\cosh (k n))^{2}}\right)$ 


$$
\left(\frac{1}{\cosh (k n+k)}\right)(\tanh (k))^{2} \ldots
$$

Hence, the solution of the original problem is given by

$$
\begin{aligned}
\mathrm{u}_{\mathrm{n}}= & \tanh (\mathrm{k}) \tanh (\mathrm{kn})+\frac{1}{\Gamma(\alpha+1)} \mathrm{t}^{\alpha}(\cosh (2 \mathrm{k})+\cosh (2 \mathrm{kn})) \\
& \left(\frac{1}{\cosh (k n-k)}\right)\left(\frac{1}{(\cosh (k n))^{2}}\right) \\
& \left(\frac{1}{\cosh (k n+k)}\right)(\tanh (k))^{2} \ldots
\end{aligned}
$$

Table 2: Numerical comparison of problem 2 for $k=0.1 t=0.01$

\begin{tabular}{|c|c|c|c|c|c|c|c|}
\hline $\mathrm{n}$ & $\alpha=0.5$ & & $\alpha=0.75$ & & & $\alpha=1$ & \\
\hline & $\mathrm{SA}$ & ADTM & SA & ADTM & SA & ADTM & Exact \\
-20 & -0.291307 & -0.291307 & -0.291308 & -0.291308 & -0.291309 & -0.291309 & -0.291309 \\
-10 & -0.289080 & -0.289134 & -0.289502 & -0.289509 & -0.289695 & -0.289694 & -0.289694 \\
0 & 0.060562 & 0.059690 & 0.032840 & 0.032701 & 0.016973 & 0.0169534 & 0.0169534 \\
10 & 0.290275 & 0.290363 & 0.290150 & 0.290162 & 0.290030 & 0.290030 & 0.290030 \\
20 & 0.291310 & 0.291310 & 0.291310 & 0.29131 & 0.291309 & 0.291309 & 0.291309 \\
\hline
\end{tabular}

\section{Problem 3}

Consider the following fractional differential-difference problem

$$
D_{t}^{\alpha} U_{n}(t)=\left(u_{n}(t)\right)^{2}\left(u_{n+1}(t)-u_{n-1}(t)\right)
$$

with initial condition

$$
\mathrm{u}_{\mathrm{n}}(0)=1-\frac{1}{\mathrm{n}^{2}}
$$

The exact solution of Eq. (55) for $\alpha=1$ can be written as:

$$
u_{n}(t)=1-\frac{1}{(n+2 t)^{2}}
$$

By the sensitivity approach and ADTM the solution of the problem are given by

$$
\begin{aligned}
& u_{n}(t)=1-\frac{1}{n^{2}}+\frac{4 t^{\alpha}}{n^{3} \Gamma(\alpha+1)}-\frac{24 t^{2} \alpha}{n^{4} \Gamma(2 \alpha+1)}+\ldots \\
& u_{n}(t)=1-\frac{1}{n^{2}}+\frac{4 t^{\alpha}}{n^{3} \Gamma(\alpha+1)}-\frac{12 t^{2} \alpha}{n^{4}(\Gamma(\alpha+1))^{2}}+\ldots
\end{aligned}
$$


Table 3: Numerical comparison of problem 3 at $t=0.3$

\begin{tabular}{|c|c|c|c|c|c|c|c|}
\hline $\mathrm{n}$ & $\alpha=0.5$ & & $\alpha=0.75$ & & & $\alpha=1$ & \\
\hline & SA & ADTM & SA & ADTM & SA & ADTM & Exact \\
-20 & 0.997137 & 0.99716 & 0.997259 & 0.997264 & 0.997343 & 0.997343 & 0.997343 \\
-10 & 0.986425 & 0.98698 & 0.987878 & 0.987971 & 0.988683 & 0.988683 & 0.988683 \\
10 & 0.991922 & 0.992079 & 0.991510 & 0.991555 & 0.988683 & 0.991100 & 0.991100 \\
20 & 0.99777 & 0.997783 & 0.997707 & 0.997707 & 0.997644 & 0.997644 & 0.997644 \\
\hline
\end{tabular}

\section{Conclusions}

In this study, we extend the sensitivity approach for solving the fractional differential difference equation and proposed a new Adomian decomposition transformation method, and obtain the analytical solution of Volterra and mKDV lattice equations. The solution shows that, both techniques are quite useful for solving a variety of linear and nonlinear fractional problems, but ADTM provides an easy and reliable scheme to be implemented on various problems. The comparison has also been done which is almost approximate to the exact solution. Numerical examples show that the suggested scheme is clearly quite efficient and potent technique in finding the solutions of the proposed equations (see table 1-3).

\section{References}

[1] E. Fermi, J. Pasta, S. Ulam, Collected Papers of Enrico Fermi, Chicago Press, volume 2, page 978, Chicago Press, 1965.

[2] P. Marquié, J. M. Bilbault, M. Rernoissnet, Observation of nonlinear localized modes in an electrical lattice, Phys. Rev. E 51 (1995), 61276133.

[3] A. S. Davydov, The theory of contraction of proteins under their excitation, J. Theoret. Biol., 38 (1973), 559-569.

[4] I. Podlubny, Fractional Differential Equations, Academic Press, San Diego - New York - London - Tokyo - Toronto, 1999.

[5] J. T. Machado, V. Kiryakova, F. Mainardi, Recent history of fractional calculus, Commun. Nonlinear Sci. Numer. Simul. 16 (2011), 1140-1153. 
[6] J. Sabatier, O. P. Agrawal, J. A. Tenreiro Machado, Advances in Fractional Calculus, Springer, Netherland, 2007.

[7] L. Decreusefond, A. S. Ustunel, Stochastic analysis of the fractional Brownian motion, Potential Anal., 10 (1999), 177-214.

[8] T. E. Duncan, Y. Hu, B. Pasik-Duncan, Stochastic calculus for fractional Brownian motion, I. Theory, SIAM J. Control Optim., 38 (2000), 582612.

[9] M. S. El Naschie, A review of E infinity theory and the mass spectrum of high energy particle physics, Chaos Solitons Fractals, 19 (2004), 209-236.

[10] G. Jumarie, A Fokker-Planck equation of fractional order with respect to time, J. Math. Phys., 33 (10) (1992), 3536-3542.

[11] E. Barkai, Fractional Fokker-Planck equation, solutions and applications, Phys. Rev. E, 63 (2001), 1-17.

[12] V. V. Anh, N. N. Leonenko, Scaling laws for fractional diffusion-wave equations with singular initial data, Statist. Probab. Lett., 48 (2000), $239-252$.

[13] A. Hanygad, Multidimensional solutions of time-fractional diffusion-wave equations, Proc. R. Soc. Lond. Ser. A Math. Phys. Eng. Sci. A 458 (2002), 933-957.

[14] S. Abbasbandy, Homotopy perturbation method for quadratic Riccati differential equation and comparison with Adomians decomposition method, Appl. Math. Comput., 172 (1) (2006), 485-490.

[15] Z. Odibat, S. Momani, Modified homotopy perturbation method: application to quadratic Riccati differential equation of fractional order, Chaos Solitons Fractals, 36 (1) (2008), 167-174.

[16] N. A. Khan, A. Ara, M. Jamil, An efficient approach for solving the Riccati equation with fractional orders, Comput. Math. Appl., 61 (2011), 2683-2689.

[17] N. A. Khan, M. Jamil, A. Ara, N. U. Khan, On efficient method for system of fractional differential equations, Adv. Differential Equations, 2011 Article ADE/303472. 
[18] J. Cang, Y. Tan, H. Xu, S. Liao, Series solutions of non-linear Riccati differential equations with fractional order, Chaos Solitons Fractals, 40 (2009), 1-9.

[19] Y. Tan, S. Abbasbany, Homotopy analysis method for quadratic Riccati differential equation, Commun. Nonlinear Sci. Numer. Simul., 13 (2008), $539-546$.

[20] M. Gülsu, M. Sezer, On the solution of the Riccati equation by the Taylor matrix method, Appl. Math. Comput., 176 (2) (2006), 414-421.

[21] G. Darmani, S. Setayeshi, H. Ramezanpour, Toward Analytic Solution of Nonlinear Differential Difference Equations via Extended Sensitivity Approach, Commun. Theor. Phys. (Beijing), 57 (1) (2012), 5-9.

[22] G. Y. Tang, Z. W. Luo, Suboptimal control of linear systems with state time-delay, In: Systems, Man, and Cybernetics, 1999. IEEE SMC'99 Conference Proceedings, 99 (5) (1999), 104-109.

[23] M. Malek-Zavarei, M. Jamshidi, Time-Delay Systems: Analysis, Optimization and Applications, North-Holland Systems and Control Series, Elsevier Science Ltd, 1987.

[24] J. H. He, Z. B., Li, Applications of the franctional complex transform to franctional differential equations, Nonlinear Science Letters A, 2 (2011), 121-126.

[25] J. H. He, S. K. Elagan, Z. B. Li, Geometrical explanation of the fractional complex transform and derivative chain rule for fractional calculus, Phys. Lett. A, 376 (2012), 257-259.

[26] J. S. Duan, An efficient algoritm for the multivariable adomian polynomials, Appl. Math. Comput., 217 (2010), 2456-2467.

[27] J. S. Duan, Convenienet analytic recurrence algorithms for the adomian polynomials, Appl. Math. Comput., 217 (2011), 6337-6348. 